\title{
Influência do índice de massa corporal e da circunferência abdominal na pressão arterial sistêmica de crianças
}

\author{
Influence of body mass index and abdominal circumference on children's systemic blood pressure
}

\author{
Marcelo Nunes lampolsky ${ }^{1}$, Fabíola Isabel S. de Souza², Roseli Oselka S. Sarni
}

\section{RESUMO}

Objetivo: Avaliar os níveis pressóricos em crianças e relacioná-los ao índice de massa corporal e à circunferência abdominal.

Métodos: Por meio de estudo prospectivo e transversal, avaliaram-se 1.408 escolares com idade entre cinco anos e dez anos e 11 meses, matriculados em escolas públicas do Município de Santo André. Foram coletados: peso ao nascer; peso e estatura, expressos como escore $Z$ do índice de massa corporal (ZIMC) e estatura para idade (ZEI). A pressão arterial (medida única) foi aferida pelo mesmo examinador. Considerou-se: desnutrição quando $Z \mathrm{IMC}<-2$, obesidade ZIMC $>+2$, baixa estatura se $Z E I<-2$; circunferência abdominal aumentada (ponto de corte $>$ P90 para sexo e idade) e pressão arterial elevada quando superior ao percentil 90 para sexo, idade e estatura. A análise estatística incluiu o teste do qui-quadrado e o cálculo da $0 d d s$ Ratio, adotando-se como significante o valor de $p<0,05$.

Resultados: A mediana de idade foi de sete anos, sendo $51 \%$ do sexo feminino. Níveis elevados foram encontrados em 19 e $12 \%$ dos escolares para a pressão arterial sistólica e diastólica, respectivamente. Verificaram-se: baixa estatura, desnutrição, obesidade e aumento da circunferência abdominal em $2,6 \%, 3,1 \%, 7,3 \%$ e $13,4 \%$ da amostra, respectivamente. A presença de obesidade foi o fator mais fortemente associado ao aumento de pressão arterial sistólica (OR 2,1; IC95\% 1,3-3,3; $p<0,001$ ) e diastólica (OR 2,6; IC95\% $1,6-4,3 ; p<0,001)$. A circunferência abdominal também se associou com pressão arterial sistólica elevada (OR 1,6; IC95\% 1,0-2,5; $p=0,027)$.
Conclusões: A pressão arterial sistêmica elevada em crianças associa-se à obesidade e ao aumento da circunferência abdominal.

Palavras-chave: obesidade; circunferência abdominal; hipertensão; índice de massa corporal; criança.

\section{ABSTRACT}

Objective: To evaluate blood pressure levels in children, relating them to body mass index and abdominal circumference.

Methods: This cross-sectional prospective study enrolled 1.408 school children, aged between five and ten years and 11 months, in the municipality of Santo Andre, São Paulo, Brazil. The following variables were evaluated: birth weight, weight and height, expressed as body mass index $\mathrm{Z}$ score (ZBMI) and height to age $\mathrm{Z}$ score $(\mathrm{ZH})$, and waist circumference (WC). Blood pressure was measured once by the same physician. Malnutrition was considered when ZBMI $<-2$, obesity when $\mathrm{ZBMI}>+2$, short stature when $\mathrm{ZH}<-2$, increased abdominal circumference when $>$ P90 for age and gender, and increased blood pressure when > P90 for age, gender and height. Statistical analysis included chi-square test and Odds Ratio, being significant $\mathrm{p}<0.05$.

Results: Mean age was seven years old, and 51\% were females. High systolic blood pressure levels were observed in $19 \%$ and elevated diastolic blood pressure in $12 \%$. Short stature, malnutrition, obesity and increased abdominal circumference were diagnosed in $2.6 \%, 3.1 \%, 7.3 \%$ and $13.4 \%$, respectively, of the studied population. The presence
Instituição: Departamento de Pediatria da Faculdade de Medicina do ABC (FMABC), Santo André, SP, Brasil

${ }^{1}$ Médico da Disciplina de Hebiatria do Departamento de Pediatria da FMABC, Santo André, SP, Brasil

${ }^{2}$ Mestre; Médica Colaboradora do Serviço de Nutrologia do Departamento de Pediatria da FMABC, Santo André, SP, Brasil

${ }^{3}$ Doutora; Professora Assistente e Coordenadora do Serviço de Nutrologia do Departamento de Pediatria da FMABC; Médica Assistente do Departamento de Pediatria da Escola Paulista de Medicina da Universidade Federal de São Paulo, São Paulo, SP, Brasil
Endereço para correspondência:

Roseli Oselka Saccardo Sarni

Rua René Zamlutti, 94, apto. 52 - Vila Mariana

CEP 04116-260 - São Paulo/SP

E-mail: rssarni@uol.com.br

Conflito de interesses: nada a declarar

Recebido em: 8/3/09

Aprovado em: 3/9/09 
of obesity was strongly associated with high systolic (OR $2.1,95 \%$ IC $1.3-3.3 ; p<0.001)$ and diastolic blood pressure (OR 2.6, 95\% IC 1.6-4.3; $p<0.001)$. Increased abdominal circumference was also an important risk factor for high systolic blood pressure (OR 1.6; 95\% IC 1.0-2.5; $p=0.027$ ).

Conclusions: High blood pressure in children is associated with obesity and increased abdominal circumference.

Key-words: obesity; abdominal circumference; hypertension; body mass index; child.

\section{Introdução}

A hipertensão arterial é uma das doenças crônicas mais prevalentes em adultos no Brasil e em outros países do mundo ${ }^{(1)}$. Em crianças, a hipertensão primária é rara, entretanto cresce a cada dia o número de indivíduos portadores de pressão arterial elevada secundária a doenças como a obesidade. A obesidade aumenta em três vezes o risco de pressão arterial elevada em comparação a crianças com peso corporal adequado ${ }^{(2)}$.

Recomenda-se que toda criança acima de três anos tenha sua pressão arterial (PA) aferida rotineiramente na consulta pediátrica, sendo o método preferencial o auscultatório. Especial atenção deve ser dispensada ao tamanho adequado do manguito para a correta aferição, salientando-se que os valores elevados devem ser confirmados em visitas repetidas. Nas crianças com idade inferior a três anos, recomenda-se avaliar a PA em todas as consultas médicas nas seguintes condições: prematuridade ou recém-nascido de muito baixo peso; permanência em unidade de terapia intensiva neonatal; cardiopatias congênitas; infecção urinária de repetição, hematúria ou proteinúria; malformações ou doenças nefro-urológicas; história familiar de doença renal congênita; transplante de órgãos sólidos ou de medula; uso crônico de medicações com efeito na pressão arterial; doenças sistêmicas que cursam com hipertensão (por exemplo, neurofibromatose e esclerose tuberosa) e risco de aumento da pressão intracraniana.

A fisiopatologia das modificações na PA atribuídas à elevação do peso corporal em crianças ainda não está totalmente elucidada. Em adultos, há três mecanismos bem estabelecidos envolvidos: resistência à insulina/hiperinsulinemia; hiperatividade do sistema nervoso simpático e alterações na estrutura e função vascular ${ }^{(3)}$. Adiposidade central e resistência insulínica também foram apontadas, em estudo recente, como determinantes da pressão arterial elevada em crianças $^{(4)}$.
O excesso de gordura abdominal está associado à elevação do risco de desenvolvimento de doenças crônicas, especialmente as cardiovasculares. A medida da circunferência abdominal guarda boa relação com a gordura visceral, sendo a cintura aumentada (acima de $80 \mathrm{~cm}$ em mulheres e $94 \mathrm{~cm}$ em) um dos fatores que compõem a síndrome metabólica em adultos $^{(4)}$. Estudo populacional americano indicou que a elevação da medida de circunferência abdominal em crianças e adolescentes associa-se de forma independente com alterações do perfil lipídico, hiperinsulinismo (mecanismo envolvido na gênese da hipertensão) e resistência insulínica ${ }^{(5)}$.

É muito importante a inclusão da aferição da pressão arterial na rotina do exame clínico em Pediatria, possibilitando intervenção precoce, visto que seus níveis elevados na infância estão associados com sua persistência na vida adulta e consequente elevação no risco cardiovascular ${ }^{(6)}$. Estudos ressaltam a relação entre os níveis pressóricos elevados em crianças e o risco para desenvolvimento de hipertensão e síndrome metabólica no futuro ${ }^{(7)}$. O risco relativo para o desenvolvimento dessas doenças na vida adulta eleva-se com o número de medidas alteradas durante a infância e adolescência, ressaltando-se a importância de detecção e intervenção precoces. Infelizmente, a prática de aferição da pressão arterial ainda não foi plenamente incorporada pelos pediatras $^{(2,6)}$.

O objetivo deste estudo foi avaliar, por meio de medida única, os níveis pressóricos em uma amostra de crianças do Município de Santo André e relacioná-los com o índice de massa corporal e com a circunferência abdominal dos pacientes analisados.

\section{Métodos}

Foram avaliados, por meio de estudo transversal com coleta prospectiva de dados, os alunos matriculados em três escolas públicas de bairros de baixo nível socioeconômico da cidade de Santo André, entre cinco anos a dez anos e 11 meses (pré-escolares e escolares). As escolas foram escolhidas por critério de conveniência: Escola Municipal de Ensino Infantil e Fundamental Maria Delfina $(\mathrm{n}=450)$ e Gonzaguinha $(\mathrm{n}=170)$ e Escola Estadual Professor Augusto Leite Franco ( $\mathrm{n}=1.018)$.

As avaliações foram realizadas pelo Serviço de Crescimento, Desenvolvimento e Terapia Nutricional do Departamento de Pediatria da Faculdade de Medicina do ABC como parte de um projeto longitudinal de identificação de distúrbios nutricionais e comorbidades associadas à 
obesidade em crianças do Município de Santo André. O projeto foi aprovado pelo Comitê de Ética em Pesquisa da Instituição, sendo solicitado o consentimento das escolas e dos responsáveis pelas crianças para a inclusão no estudo. Os responsáveis pelos alunos foram convocados para uma reunião com os pesquisadores na própria escola, quando foram explicados os objetivos e os procedimentos a serem realizados durante o estudo, foi solicitado o consentimento para participação e, em caso positivo, aplicado um questionário pré-codificado.

Os critérios de inclusão no estudo foram: idade do escolar entre cinco anos e dez anos e 11 meses, frequência regular à escola e ausência de caracteres sexuais secundários (avaliados por examinador único - médico pediatra, segundo Tanner $)^{(8)}$. Como critérios de exclusão, citam-se: presença de doença crônica (endócrina, infecciosa, cardíaca ou renal) ou genética, doença aguda no momento do estudo, uso de medicamentos imunossupressores, anti-hipertensivos ou diuréticos.

Preencheram os critérios de inclusão 1.408 pré-escolares e escolares que compuseram a casuística do estudo. Por 15 dias consecutivos, eles foram avaliados na própria escola durante os períodos da aula (manhã e tarde). As crianças foram previamente informadas sobre os procedimentos a serem realizados e encaminhadas pela professora, em grupos de cinco, para uma sala montada para obter os dados abaixo citados.

O peso e a estatura foram avaliados de acordo com a recomendação da Organização Mundial da Saúde (OMS), em $1995^{(9)}$. A circunferência abdominal foi obtida no ponto médio entre a décima costela e a borda superior da crista ilíaca, conforme preconizado por Freedman et $a^{(5)}$.

A partir dos dados de peso e estatura, foram calculados os indicadores na forma de escore $Z$ do índice de massa corporal (ZIMC) e estatura para idade $(\mathrm{ZEI})^{(9)}$. O referencial adotado foi o CDC-2000 ${ }^{(10,11)}$. Definiu-se a presença de obesidade e desnutrição, respectivamente, nas crianças que apresentavam dois desvios padrões acima (+2Z) e abaixo (-2Z) da população de referência em relação ao ZIMC. O mesmo ponto de corte foi utilizado para classificar a baixa estatura (-2Z) em relação ao ZEI. A circunferência abdominal foi classificada segundo o sexo e a idade, sendo elevada quando a medida situava-se acima do percentil 90 em relação ao gênero e idade ${ }^{(5)}$.

A aferição e a classificação da pressão arterial sistêmica foram obtidas por meio de medida única, de um mesmo examinador, com esfigmomanômetro aneroide $\left(\operatorname{Tycos}^{\circledR}\right.$ ) padrão, previamente calibrado, segundo preconizado ${ }^{(12)}$. Foram utilizados manguitos de largura e comprimento adequados, com a câmara de ar ocupando $40 \%$ do perímetro do braço, medido a um ponto médio entre o olécrano e acrômio, cobrindo 80 a 100\% do perímetro do braço. Os níveis pressóricos foram classificados individualmente em percentis em relação ao referencial americano para sexo, idade e estatura, segundo preconização do National High Blood Pressure Education Program Working Group on High Blood Pressure in Children and Adolescents (NHBPEP) ${ }^{(12)}$. Foram considerados elevados os níveis pressóricos superiores ao percentil $90^{(12)}$.

$\mathrm{Na}$ análise estatística, as variáveis foram testadas quanto à sua normalidade por meio do teste de KolmogorovSmirnov. Utilizaram-se as médias e as medianas como medidas de tendência central para as variáveis contínuas paramétricas e não-paramétricas, respectivamente. A PA sistólica e diastólica foram descritas para sexo e idade (média, mediana e desvio padrão), ajustadas para a estatura. Utilizou-se o teste qui-quadrado e o cálculo do Odds Ratio para avaliar a associação da PA com as variáveis estudadas. Considerou-se 5\% o nível de significância para rejeição da hipótese de nulidade

\section{Resultados}

Quanto às características gerais dos 1.408 pré-escolares e escolares, a mediana de idade da população estudada foi 7,2 anos (variação: 5,0-10,9 anos), sendo $712(50,6 \%)$ do sexo feminino. $\mathrm{O}$ baixo peso ao nascer foi referido em 125 $(10,7 \%)$ escolares (Tabela 1$)$.

Em relação à condição nutricional, observou-se baixa estatura em 36 (2,6\%), desnutrição em $43(3,1 \%)$ e obesidade em $103(7,3 \%)$ escolares (Tabela 1). A circunferência abdominal acima do percentil 90 foi encontrada em 140 $(13,4 \%)$ crianças. Níveis pressóricos elevados (acima do percentil 90 para sexo e idade) foram observados em 270 $(19,2 \%)$ e $172(12,2 \%)$ escolares para a pressão arterial sistólica e diastólica, respectivamente (Tabela 1). A Tabela 2 expressa a média, a mediana, o desvio padrão e os percentis 90 e 95 da pressão arterial sistólica e diastólica dos pacientes avaliados, agrupados por faixa etária e sexo, ajustado para a estatura.

Presença de obesidade (ZIMC>2) foi o fator mais fortemente associado ao aumento de pressão arterial sistólica (OR 2,1; $p<0,001)$ e diastólica (OR 2,6; $p<0,001$ ), respectivamente (Tabela 3 ). A circunferência abdominal também se mostrou um fator de risco importante para pressão arterial sistólica elevada (OR 1,6; $p=0,027)$. 
Tabela 1 - Caracterização dos 1.408 pré-escolares e escolares estudados

\begin{tabular}{|c|c|c|}
\hline Variável & $\mathbf{n}$ & $\%$ \\
\hline \multicolumn{3}{|l|}{ Escola } \\
\hline Maria Delfina & 354 & 25,1 \\
\hline Leite Franco & 947 & 67,3 \\
\hline Gonzaguinha & 107 & 7,5 \\
\hline \multicolumn{3}{|l|}{ Sexo } \\
\hline Feminino & 712 & 50,6 \\
\hline Masculino & 696 & 49,4 \\
\hline \multicolumn{3}{|l|}{ Idade } \\
\hline 5-7 anos & 344 & 24,4 \\
\hline $7-10$ anos & 890 & 63,2 \\
\hline$>10$ anos & 174 & 12,4 \\
\hline \multicolumn{3}{|l|}{ Peso ao nascer } \\
\hline$<2500 \mathrm{~g}$ & 125 & 10,7 \\
\hline$\geq 2500 \mathrm{~g}$ & 1.047 & 89,3 \\
\hline \multicolumn{3}{|c|}{ Cintura abdominal } \\
\hline 2P90 & 140 & 13,3 \\
\hline$<\mathrm{P} 90$ & 907 & 86,6 \\
\hline \multicolumn{3}{|c|}{ Pressão arterial sistólica } \\
\hline$<\mathrm{P} 90$ & 1.138 & 80,8 \\
\hline$\geq$ P90 e <P95 & 117 & 8,4 \\
\hline$\geq$ P95 e <P99 & 108 & 7,8 \\
\hline 2P99 & 45 & 3,2 \\
\hline \multicolumn{3}{|c|}{ Pressão arterial diastólica } \\
\hline$<\mathrm{P} 90$ & 1.236 & 87,8 \\
\hline$\geq$ P90 e <P95 & 89 & 6,3 \\
\hline$\geq$ P95 e <P99 & 71 & 5,0 \\
\hline 2P99 & 12 & 0,9 \\
\hline \multicolumn{3}{|l|}{ Z escore El } \\
\hline$\leq-2$ & 36 & 2,6 \\
\hline$-2 a-1$ & 194 & 13,8 \\
\hline$>-1$ & 1.178 & 83,7 \\
\hline \multicolumn{3}{|l|}{ Z escore IMC } \\
\hline$\leq-2$ & 43 & 3,1 \\
\hline$-2 a-1$ & 147 & 10,4 \\
\hline-1 a 1 & 852 & 60,5 \\
\hline 1 a 2 & 263 & 18,7 \\
\hline$>2$ & 103 & 7,3 \\
\hline
\end{tabular}

EI: estatura para idade; IMC: índice de massa corporal.

\section{Discussão}

A hipertensão arterial é indiscutivelmente um importante fator de risco para o desenvolvimento de doenças cardiovasculares em adultos, contribuindo para 69\% dos acidentes vasculares cerebrais e $49 \%$ das doenças coronarianas $^{(13)}$. Na infância, geralmente é assintomática e sua descoberta é com frequência casual ou dependente de suas complicações.
A prevalência de hipertensão na infância, relatada em diversos estudos nacionais e internacionais, varia amplamente de 1,2 a $13 \%$, dependendo, sobretudo, da metodologia empregada, da faixa etária estudada e do número de medidas aferidas $^{(12-18)}$. Em Maceió (AL), utilizando-se duas aferições de pressão arterial em 1.253 alunos, 9,4\% deles apresentavam PA acima do percentil $95^{(16)}$. No presente trabalho, obteve-se prevalência de 10 e $6 \%$ de escolares com medida única de pressão arterial sistólica e diastólica, respectivamente, acima do percentil 95. Embora seja recomendada a obtenção de três medidas seriadas de PA, em estudos epidemiológicos pode ser utilizada a medida única como rastreamento, desde que realizada pelo mesmo examinador, com rigor nas técnicas empregadas. Os casos suspeitos detectados devem ser encaminhados a serviços de saúde para confirmação ${ }^{(19)}$. No presente estudo, as crianças com valores de pressão arterial alterada foram encaminhadas por meio de formulário padrão para aferição de mais duas medidas de pressão arterial na unidade básica de saúde mais próxima ao domicílio para confirmação diagnóstica.

O baixo peso ao nascer é um dos fatores considerados de risco para futuras elevações nos níveis pressóricos na infância e na vida adulta ${ }^{(20)}$. Entretanto, outros fatores como o rápido e acentuado ganho de peso no primeiro ano de vida, por vezes mais frequente em recém-nascidos com baixo peso, têm sido relatados como de grande importância na gênese da hipertensão na vida adulta ${ }^{(21)}$. Os resultados encontrados no presente estudo não encontraram relação entre baixo peso ao nascer com níveis elevados de pressão arterial. Esse fato pode ser explicado pela característica multifatorial da hipertensão e por ser o dado de peso ao nascer referido pela mãe, portanto sujeito a viés de informação.

A distribuição da pressão arterial por sexo e idade e ajustada para a estatura (Tabela 2) permite observar que a PA, especialmente a sistólica, aumenta com a idade em ambos os sexos (associação com o aumento de massa corporal). A puberdade, em estudo recente, foi associada ao aumento da pressão arterial, sendo tal associação relacionada aos hormônios gonadais, com efeito preponderante da testosterona ${ }^{(22)}$.

A hipertensão primária é pouco frequente em crianças, entretanto, cresce a cada dia o número de indivíduos portadores de pressão arterial elevada secundária a hábitos alimentares e estilo de vida inadequado e a doenças como a obesidade. No Brasil, verifica-se um processo de transição nutricional, nas últimas décadas, traduzido pela elevação na prevalência de sobrepeso e obesidade e redução nas taxas de desnutrição em crianças e adolescentes. A Pesquisa de Orçamentos Fa- 
Tabela 2 - Distribuição das medidas de pressão arterial sistólica e diastólica em meninos e meninas, ajustada para estatura

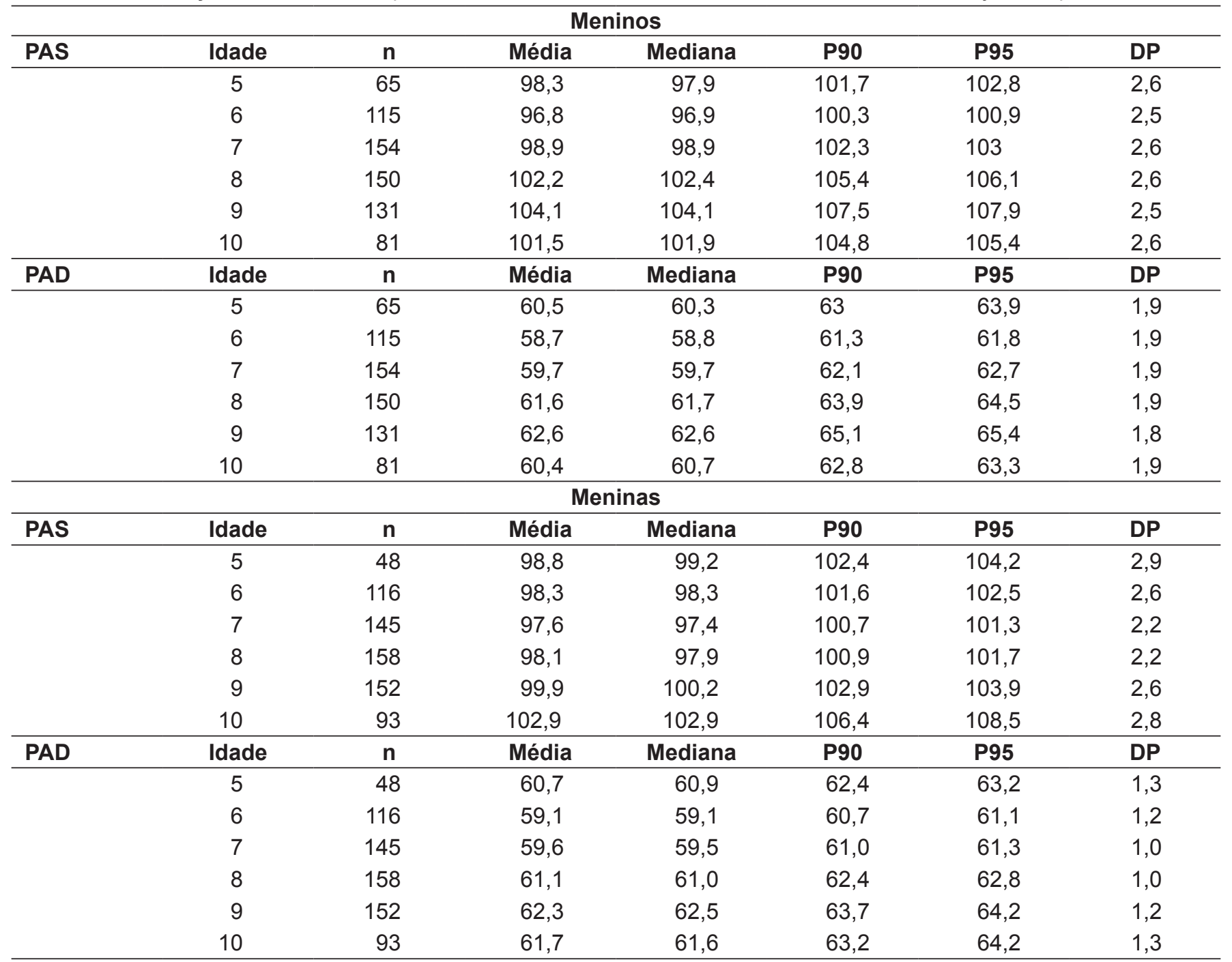

PAS: pressão arterial sistólica; PAD: pressão arterial diastólica; DP: desvio padrão.

Tabela 3 - Odds Ratio da pressão arterial elevada em relação ao baixo peso ao nascer, circunferência abdominal aumentada (CA) e presença de obesidade

\begin{tabular}{|c|c|c|c|c|c|c|c|c|c|}
\hline \multirow{2}{*}{ Variável } & & \multicolumn{4}{|c|}{ PA sistólica } & \multicolumn{4}{|c|}{ PA diastólica } \\
\hline & & $\begin{array}{l}\geq \mathrm{P} 90 \\
\mathrm{n}=270\end{array}$ & $\begin{array}{c}<\text { P90, } \\
n=1138\end{array}$ & $\begin{array}{c}\text { OR } \\
\text { (IC 95\%) }\end{array}$ & $p$ & $\begin{array}{l}\geq P 90, \\
n=172\end{array}$ & $\begin{array}{c}<\mathrm{P} 90 \\
\mathrm{n}=1236\end{array}$ & $\begin{array}{c}\text { OR } \\
\text { (IC 95\%) }\end{array}$ & $p$ \\
\hline PN & $<2500 \mathrm{~g}$ & $\begin{array}{c}28 \\
(10,3 \%)\end{array}$ & $\begin{array}{c}97 \\
(8,5 \%)\end{array}$ & $\begin{array}{c}1,3 \\
(0,8-2,1)\end{array}$ & 0,214 & $\begin{array}{c}19 \\
(11 \%)\end{array}$ & $\begin{array}{c}106 \\
(8,6 \%)\end{array}$ & $\begin{array}{c}1,4 \\
(0,8-2,5)\end{array}$ & 0,162 \\
\hline Sexo & Masculino & $\begin{array}{c}140 \\
(51,8 \%)\end{array}$ & $\begin{array}{c}549 \\
(48,2 \%)\end{array}$ & $\begin{array}{c}1,1 \\
(0,9-1,5)\end{array}$ & 0,295 & $\begin{array}{c}92 \\
(53,5 \%)\end{array}$ & $\begin{array}{c}594 \\
(48,0 \%)\end{array}$ & $\begin{array}{c}1,1 \\
(0,8-1,5)\end{array}$ & 0,619 \\
\hline $\mathrm{CA}$ & >P90 & $\begin{array}{c}33 \\
(12,2 \%)\end{array}$ & $\begin{array}{c}107 \\
(9,4 \%)\end{array}$ & $\begin{array}{c}1,6 \\
(1,0-2,5)\end{array}$ & 0,027 & $\begin{array}{c}18 \\
(10,5 \%)\end{array}$ & $\begin{array}{c}122 \\
(9,9 \%)\end{array}$ & $\begin{array}{c}1,3 \\
(0,7-2,3)\end{array}$ & 0,365 \\
\hline Z escore IMC & $\geq 2$ & $\begin{array}{c}33 \\
(12,2 \%)\end{array}$ & $\begin{array}{c}70 \\
(6,1 \%)\end{array}$ & $\begin{array}{c}2,1 \\
(1,3-3,3)\end{array}$ & $<0,001$ & $\begin{array}{c}26 \\
(15,1 \%)\end{array}$ & $\begin{array}{c}77 \\
(6,2 \%)\end{array}$ & $\begin{array}{c}2,6 \\
(1,6-4,3)\end{array}$ & $<0,001$ \\
\hline $\mathrm{ZIMC}+\mathrm{CA}$ & $\geq 2+>P 90$ & $\begin{array}{c}19 \\
(7,0 \%)\end{array}$ & $\begin{array}{c}44 \\
(3,9 \%)\end{array}$ & $\begin{array}{c}2,2 \\
(1,2-4,0)\end{array}$ & 0,004 & $\begin{array}{c}12 \\
(7,0 \%)\end{array}$ & $\begin{array}{c}51 \\
(4,1 \%)\end{array}$ & $\begin{array}{c}2,1 \\
(1,0-4,2)\end{array}$ & 0,026 \\
\hline
\end{tabular}

PA: pressão arterial; PN: peso ao nascer; CA: circunferência abdomina; IMC: índice de massa corporal. 
miliares (POF) 2002-2003 realizada pelo Instituto Brasileiro de Geografia e Estatística (IBGE) demonstrou prevalência de sobrepeso e obesidade em 20 e $18 \%$ dos adolescentes brasileiros de sexo masculino e feminino, respectivamente ${ }^{(23)}$. Além da expressiva ascensão na prevalência de obesidade, chama a atenção o aparecimento precoce de formas graves, com comorbidades associadas. Estudo conduzido por Ferreira et al ${ }^{(24)}$ em 1.550 escolares, em Brasília, encontrou 17,3\% de prevalência de síndrome metabólica, sendo que $15,2 \%$ das crianças avaliadas eram hipertensas. A prevalência de crianças obesas (ZIMC $\geq 2$ ) na amostra estudada foi de 7,3\%, valor semelhante ao observado em estudo conduzido com escolares em Florianópolis ${ }^{(25)}$, que identificou prevalência de sobrepeso e obesidade de 17,9 e $6,7 \%$, respectivamente.

Estudo epidemiológico evidenciou que a obesidade eleva cerca de três vezes o risco de pressão arterial elevada em comparação a crianças com peso corporal adequado ${ }^{(2)}$. O escore $\mathrm{Z}$ do IMC no presente estudo revelou-se um fator de risco fortemente associado à elevação da pressão arterial sistólica e diastólica. As crianças com ZIMC $\geq 2$ apresentaram duas vezes mais chances de desenvolverem PA sistólica e diastólica elevada. A fisiopatologia das modificações na PA atribuídas à elevação do peso corporal ainda não está totalmente elucidada. Estudos em crianças que investigam a associação de obesidade e hipertensão têm ressaltado a participação de distúrbios na função autonômica, resistência insulínica e anormalidades na função e estrutura vascular ${ }^{(2)}$. O Bogalusa Heart Study corrobora a hipótese da hiperatividade autonômica ao relatar que a frequência cardíaca em repouso correlaciona-se positivamente com a pressão arterial e com as medidas de adiposidade em crianças e adolescentes ${ }^{(26)}$.

A relação entre resistência insulínica e hipertensão se torna mais evidente em estudos que envolvem obesos, sugerindo o papel adicional de fatores diretamente relacionados ao tecido adiposo. Os adipócitos produzem uma ampla gama de peptídeos vasoativos (TNF-alfa, angiotensinogênio) que podem prejudicar os efeitos vasodilatadores da insulina ${ }^{(27)}$. Vários fatores são apontados no desenvolvimento da hipertensão associada à resistência insulínica. O hiperinsulinismo e a resistência insulínica podem levar à retenção renal de sódio, reduzindo a natriurese e contribuindo para a elevação da PA. Outra hipótese é que a hiperinsulinemia estimule o sistema nervoso simpático, induzindo a vasoconstricção, elevando o débito cardíaco e aumentando a reabsorção renal de sódio, todos mecanismos envolvidos na elevação da $\mathrm{PA}^{(27)}$.

A insulina é um potente vasodilatador e influencia diretamente a função endotelial, o tônus vascular e o fluxo de sangue para os tecidos, o que influencia a liberação do óxido nítrico. Além disso, a insulina ativa o fator de crescimento do endotélio (FCEV) vascular. Em situações de resistência insulínica, seu efeito vasodilatador é perdido e a produção de FCEV diminui, resultando em redução na disponibilidade do óxido nítrico e consequente disfunção endotelial e hipertensão. Finalmente, o tecido adiposo contribui diretamente para a ativação do sistema renina-angiotensina-aldosterona. $\mathrm{O}$ angiotensinogênio, precursor do poderoso vasoconstrictor angiotensina II, é expresso e produzido nos adipócitos ${ }^{(27)}$. Elevação na produção do angiotensinogênio em obesos foi relacionada ao aparecimento de hipertensão ${ }^{(28)}$. A angiotensina II estimula diretamente a expressão de várias moléculas de adesão nas células vasculares e a angiogênese, além de reduzir a disponibilidade do óxido nítrico, processos esses relacionados à disfunção endotelial. Estudo realizado em crianças obesas pré-púberes observou associação positiva entre resistência insulínica e elevação de PA, avaliada por método oscilométrico com monitorização de 24 horas. As crianças com valores mais alterados de Homeostasis Model Assessment (HOMA-IR), equação para mensurar a resistência insulínica, tinham valores significativamente mais altos de $\mathrm{PA}^{(29)}$.

Em relação às anormalidades na estrutura e função vascular em crianças obesas, descreve-se na literatura a vasculopatia semelhante à de portadores de diabetes e dislipidemias. Tounian et al observaram alteração na complacência arterial, com menor distensibilidade e comprometimento na função endotelial em crianças gravemente obesas comparadas a controles ${ }^{(29)}$.

$A$ adiposidade central tem um papel bem estabelecido no risco cardiovascular e metabólico em adultos ${ }^{(30)}$ e crianças $^{(31)}$. Com respeito à elevação da $\mathrm{PA}$, a circunferência abdominal também aparece como forte preditor ${ }^{(32)}$. O Bogalusa Heart Study enfatizou a importância da distribuição de gordura corporal, especialmente a circunferência abdominal e sua relação com dislipidemia e resistência insulínica em crianças e adolescentes. Os autores verificaram maiores concentrações de colesterol LDL, triglicérides, insulina e taxas mais baixas de colesterol HDL em crianças americanas com cintura acima do percentil 90 comparadas àquelas com medida inferior ao percentil $10^{(5,10)}$. O presente estudo mostrou que as crianças com medidas de circunferência abdominal acima do percentil 90 de acordo com o referencial adotado, mostraram maior risco para pressão arterial sistólica elevada.

Os mecanismos pelos quais o tecido adiposo, especialmente o visceral, contribui para o desenvolvimento da resistência insulínica e consequente elevação da PA ainda não são conhe- 
cidos. Dois mecanismos têm sido implicados. Por um lado, há elevação dos ácidos graxos livres circulantes que estimulam a gliconeogênese e a glicogenólise, o que resulta em aumento da produção hepática de glicose e induz resistência à insulina. Por outro lado, estabelece-se um estado inflamatório, pois os ácidos graxos livres estimulam a elevação das adipocitocinas pró-inflamatórias (TNF, IL6, leptina), com redução das antiinflamatórias, como a adiponectina, e desbalanço que diminui a sensibilidade à insulina nos tecidos ${ }^{(27,28)}$.

Nesse contexto, o presente estudo reforça a contribuição da obesidade, especialmente de distribuição central, no estabelecimento de hipertensão arterial na infância.

\section{Referências bibliográficas}

1. Rodgers A, Lawes $\mathrm{C}$, MacMahon S. Reducing the global burden of blood pressure-related cardiovascular disease. J Hypertens Suppl 2000;18:S3-6.

2. Sorof J, Daniels S. Obesity hypertension in children: a problem of epidemic proportions. Hypertension 2002;40:441-7.

3. Ferrannini E, Natali A, Capaldo B, Lehtovirta M, Jacob S, Yki-Järvinen H. Insulin resistance, hyperinsulinemia, and blood pressure: role of age and obesity. European Group for the Study of Insulin Resistance (EGIR). Hypertension 1997;30:1144-9.

4. Marcovecchio ML, Patricelli LB, Zito MB, Capanna RA, Ciampani MA, Chiarelli FA et al. Ambulatory blood pressure monitoring in obese children: role of insulin resistance. J Hypertens 2006;24:2431-6.

5. Freedman DS, Serdula MK, Srinivasan SR, Berenson GS. Relation of circumferences and skinfold thickness to lipid and insulin concentrations in children and adolescents: the Bogalusa Heart Study. Am J Clin Nutr 1999;69:308-17.

6. Williams CL, Hayman LL, Daniels SR, Robinson TN, Steinberg J, Paridon S et al. Cardiovascular health in childhood: A statement for health professionals from the Committee on Atherosclerosis, Hypertension, and Obesity in the Young (AHOY) of the Council on Cardiovascular Disease in the Young, American Heart Association. Circulation 2002;106:143-60.

7. Bao W, Threefoot SA, Srinivasan SR, Berenson GS. Essential hypertension predicted by tracking of elevated blood pressure from childhood to adulthood: the Bogalusa Heart Study. Am J Hypertens 1995;8:657-65.

8. Marshall WA, Tanner JM. Variations in pattern of pubertal changes in girls. Arch Dis Child 1969;44:291-303.

9. World Health Organization. Physical Status: the use and interpretation of anthropometry. WHO Technical Report Series 854. Geneva: WHO; 1995.

10. Klein S, Allison DB, Heymsfield SB, Kelley DE, Leibel RL, Nonas C et al. Waist circumference and cardiometabolic risk: a consensus statement from shaping America's health: Association for Weight Management and Obesity Prevention; NAASO, the Obesity Society; the American Society for Nutrition; and the American Diabetes Association. Diabetes Care 2007;30:1647-52.

11. Kuczmarski RJ, Ogden CL, Grummer-Strawn LM. CDC growth charts: United States. Advance data from vital and health statistics; $n^{\circ} 314$. Hyattsville, Maryland: National Center for Health Statistics, 2000.

12. National High Blood Pressure Education Program Working Group on High Blood Pressure in Children. The fourth report on the diagnosis, evaluation, and treatment of high blood pressure in children and adolescents. Pediatrics 2004;114(Suppl 2):555-76.

13. World Health Organization. The World Health Report 2002: reducing risks, promoting healthy life. Geneva, Switzerland: WHO; 2002.

14. Freitas OC, Resende de Carvalho F, Marques Neves J, Veludo PK, Silva Parreira R, Marafiotti GR et al. Prevalence of hypertension in the urban population of Catanduva, in the State of Sâo Paulo, Brazil. Arq Bras Cardiol 2001;77:9-21.

15. Garcia FD, Terra AF, Queiroz AM, Correia CA, Ramos PS, Ferreira QT et al. Evaluation of risk factors associated with increased blood pressure in children. J Pediatr (Rio J) 2004;80:29-34.
16. Moura AA, Silva MA, Ferraz MR, Rivera IR. Prevalence of high blood pressure in children and adolescents from the city of Maceió, Brazil. J Pediatr (Rio J) 2004;80:35-40.

17. Oliveira AM, Oliveira AC, Almeida MS, Almeida FS, Ferreira JB, Silva CE et al Environmental and anthropometric factors associated with childhood arterial hypertension. Arq Bras Endocrinol Metabol 2004;48:849-54.

18. Rezende DF, Scarpelli RA, de Souza GF, da Costa JO, Scarpelli AM, Scarpelli PA et al. Prevalence of systemic hypertension in students aged 7 to 14 years in the municipality of Barbacena, in the State of Minas Gerais, in 1999. Arq Bras Cardiol 2003;81:381-6.

19. Lima EM. Assessment of risk factors associated with elevated blood pressure in children and adolescents. J Pediatr (Rio J) 2004;80:3-5.

20. Lurbe E, Garcia-Vicent C, Torro I, Fayos JL, Aguilar F, Llano JM et al. First-year blood pressure increase steepest in low birthweight newborns. J Hypertens 2007;25:81-6.

21. Huxley RR, Shiell AW, Law CM. The role of size at birth and postnatal catchup growth in determining systolic blood pressure: a systematic review of the literature. J Hypertens 2000;18:815-31.

22. Shankar RR, Eckert GJ, Saha C, Tu W, Pratt JH. The change in blood pressure during pubertal growth. J Clin Endocrinol Metab 2005;90:163-7.

23. IBGE. Pesquisa de orçamentos familiares 2002-2003. Antropometria e análise do estado nutricional de crianças e adolescentes no Brasil. Rio de Janeiro: IBGE, 2004.

24. Ferreira AP, Oliveira CE, França NM. Metabolic syndrome and risk factors for cardiovascular disease in obese children: the relationship with insulin resistance (HOMA-IR). J Pediatr (Rio J) 2007;83:21-6.

25. Assis MA, Rolland-Cachera MF, Vasconcelos FA, Bellisle F, Calvo MC, Luna ME et al. Overweight and thinness in 7-9 year old children from Florianópolis, Southern Brazil: a comparison with a French study using a similar protocol. Rev Nutr 2006;19:299-308.

26. Jiang X, Srinivasan SR, Urbina E, Berenson GS. Hyperdynamic circulation and cardiovascular risk in children and adolescents. The Bogalusa Heart Study. Circulation 1995;91:1101-6.

27. Wassink AM, Olijhoek JK, Visseren FL. The metabolic syndrome: metabolic changes with vascular consequences. Eur J Clin Invest 2007;37:8-17.

28. Ahima RS, Flier JS. Adipose tissue as an endocrine organ. Trends Endocrinol Metab 2000;11:327-32.

29. Tounian P, Aggoun Y, Dubern B, Varille V, Guy-Grand B, Sidi D et al. Presence of increased stiffness of the common carotid artery and endothelial dysfunction in severely obese children: a prospective study. Lancet 2001;358:1400-4.

30. Lurbe E, Alvarez V, Rendon J. Obesity, body fat distribution and ambulatory blood pressure in children and adolescents. J Clin Hypertens 2001;3:362-7.

31. Lurbe E, Alvarez V, Liao Y, Tacons J, Cooper R, Cremades B. The impact of obesity and body fat distribution on ambulatory blood pressure in children and adolescents. Am J Hypertens 1998;11:418-24.

32. Vincent JA, Geithner CA, Cooper MF, Siegel SR, Weixel CA, Windlin EM. Fatness, fat distribution, and blood pressure: relationships, prediction, and tracking in children. Med Sci Sports Exerc 2006;38:433-4. 